\title{
Developing Cloud OS
}

\author{
Gaurav Chhatwal, Rishabh Shah, Kavita Pandey and Prakash Kumar \\ Jaypee Institute of Information Technology, Noida, 201309, India \\ E-mail: gaurav10503882@gmail.com, rishabhshah1991@gmail.com, kavita.pandey@jiit.ac.in, \\ prakash.kumar@jiit.ac.in
}

Received: 17 October 2017; Accepted: 08 January 2018; Published: 08 April 2018

\begin{abstract}
This computing which runs on a web browser. It provides access to a number of web applications through internet without booting the whole OS. The purpose behind Cloud Operating System is that the full system is running on the Web browser and lives on it. Cloud OS is thought of as a new era of an Operating System in which everything inside an Operating System can be accessed from everywhere inside a specific network. The user just need to login onto the web browser and thus can have access to his personalized web-tops where all the applications and data is stored.

In this paper it will discuss what is the difference between the Cloud OS and a simple Operating System and how the Cloud OS is developed defining all the requirements and functionalities of a cloud OS. We will also discuss in detail about the load balancing, Georeplication Data Storage and Virtualization in Cloud OS.
\end{abstract}

Index Terms-Cloud Operating System, Load Balancing, Web-top, Geo-replication, Data Storage, Virtualization, Graphical user interface, Infrastructure as a Service.

\section{INTRODUCTION}

With the increase in the use of internet and cloud computing becoming more and more popular these days, the amount of data stored on cloud is increasing which makes it difficult to store and handle it safely. With the emergence of the Cloud Computing, applications are built keeping in mind with this paradigm are providing services for a large number of users worldwide. Supporting these kinds of application needs to offer consistency in terms of data, high availability in service and scalability so as to provide good user experience. Then only the system can run successfully with no user complaints.

Now, providing the user a good experience of using the applications and scalability, use of data replication is very necessary. Data replication means, placing multiple copies of data on different servers so that it is safe and survive to any kind of failures.

But, replicating the data to all the servers all over in the world causes a lot of redundancy and wastage of space on the server. Thus, it inspires of how to maintain the replicas consistent. So, in order to overcome this we need to store data only to those servers where the user operates his data from. This means make geo-replication of data on the cloud servers.

Why geo-replication of data??

Due to the expansion of Cloud Computing, clientsare now no longer located in a single geographic location. Applications like Facebook, Google, and Amazon Services which are used by a large-scale of clients who may access their data from different geo-graphic locations. Thus replication is a method which permits placing of data copies of the user closer to them.This in turnlets the user access the data with smaller latency. So, replication is an important component of cloud computing which should be kept in mind. For example, if Google only had a datacenter on one continent of the earth then the clients of distant continents would have been experiencing high latency when accessing Google services. Therefore copies of data should be replicated geographically to the servers so that the client gets the data from the nearest server.

In order to satisfy these requirements, it is important to imply very efficient algorithms which can even improve on the consistency.Additionally, applications should be made such that it is focusing more on performance rather than consistency because an increase in the latency of operations of the application will result in the loss ofpercentage of its users. Therefore, the main aspect in developinga cloud-computing infrastructure is to find the data storage protocols that offer a good trade-offs between efficiency and consistency.

\section{RELATED WORKS}

Cloud computing infrastructure is just a web service interface to operating system virtualization. According to NIST (National Institute of Standards and Technology), "Cloud Computing is a model for enabling convenient, on demand network access to a shared pool of configurable computing resources that can be rapidly provisioned with minimal management effort or service provider interaction." A web Cloud OS is responsible for managing the cloud infrastructure. It hides the details of the cloud infrastructure from the application programmers and co-ordinates the sharing of the limited resources [2] [7].

Cloud Operating System consists of a WebTop or can say a Web Desktop which follows the cloud computing concept [5] [7]. The GUI of the WebTop is mostly scripted using PHP, XML, and JavaScript. It behaves to 
be a platform for web applications which are written using the Cloud Computing concepts. It gives a look of a Desktop environment which has applications and system utilities. It can again be accessed by the portable devices via its mobile front end. The Cloud Operating System lets the user to upload his files and work with them no matter where he is. There are number of benefits of using Cloud OS [7]:

- The Cloud OS will deliver a fully featured and functional Workspace as a Service (WaaS) that brings consumers to move their desktop experience online on any browsers [2].

- Worldwide availability of Cloud Operating Systems

- Dynamic design: The GUI can be customized as per the needs.

- Extensive Applications: Multimedia applications, Network applications, Office applications and Chat apps, all are available.

- Remote storage availability: Files can be stored over the internet through the file browser and can be edited.

Now coming onto the backend, the development of Cloud OS architecture has been a key issue. A number of key features should be embedded into the architecture for more efficient working of Cloud OS that may involve Load Balancing, Virtualization, Geo-replication Data Storage, Scheduling, Memory management [14] and more.

In this paper, it is argued for an approach to develop an efficient and more balanced architecture for Cloud OS that would resolve the following issues:

- How the user will be able to interact with the Cloud OS?

- How the storage drives on the Cloud OS will be handled?

- How the Cloud computing resources can be efficiently used and how to reach to the maximum profits?

- How to enable efficient resource sharing for applications running on different OS?

- How to avoid data loss in cloud storage increase the availability of servers?

In SECTION II, related works in this area are discussed. SECTION III discusses the proposed algorithm alongwith the components used in our architecture. Different techniques/modules that can be applied or used to resolve these issues are elaborated. Additionally, the requirements and assumptions involved in these technologies are enlisted. It is also discussed on how these techniques can change the output's efficiency. In SECTION IV, it is discussed that how these techniques altogether can give birth to a number of different designs of Cloud OS architectures for more efficient and balanced working environment.

For the user to work on the Cloud OS, the user needs an interface to interact with it. So a module is proposed that can be developed using languages such as JavaScript, CSS, and HTML etc. The module is named as a WebTop [7].

The WebTop is a virtual desktop environment running in a web browser or similar client application. The WebTop should not be confused with the actual Operating System. Its interface looks like any desktop computer only that viewed in a browser, might be a Windows design or an IOS design or any customized design of your own [5]. WebTop may consist of a video player, audio player, a text editor, MS Office, and many other web applications. It also displays the dashboard of the user specifying its memory, CPU usage etc. On the Cloud OS WebTop the web applications, cloud storage drives, configuration, settings, and access privileges reside remotely over the network. Major part of the computing takes place remotely and not on the WebTop. The WebTop is used mainly for displaying and for input purpose.

To handle the storage drives on the Cloud OS, a number of open source tools are available in the market providing Infrastructure as a Service [2]. The same can be developed ourselves which will be solving the same purpose. Also, one can go for Amazon, Rackspace, Joyent, VMWare, Microsoft and few more which are the renowned cloud IaaS providers in the world. These companies give their own platform and framework to work upon them and customize it. They are easy to use as well. Along with these there are few opensource tools like Owncloud, Cloudstack, and Openstack etc. These can also be used as an IaaS tool for our cloud storage [4]. But what it needs is to customize it own way. Even company like Rackspace is also based on the framework of Openstack.

What proposed in this paper is using of open source software as an IaaS tool. Now, the purpose of this IaaS tool is to sync the cloud drive of the user with his WebTop, so that the cloud drive can be treated as My Computer in Windows[1][3]. This cloud drive is the cloud storage tool. Thus, accessing all the files and folders of a user can be made whenever and wherever he wants. Coming to the other aspect of IaaS tool, the physical memory, allocation to the user is also done through this IaaS tool only. There would be upto certain limit of physical memory benefits given to a user as free (like 512MB RAM and 1GB of free space). But after this limit to upgrade there would be premium charges as per his requirements. So it might be a case where the end user would be getting a high speed mobile OS to work which is better than the general PC.

To enable efficient resource sharing for applications running on different OS, we can do virtualization. With help of virtualization, multiple VMs are created on a single machine and which runs different/same OS on different VMs. These VMs have the capability to share resources i.e. Memory and CPU of the machine among each other [6].

Virtualization is a key aspect of cloud computing. Without virtualization it is very difficult to provide the 
delivery of the services to the users. Because virtualization provides a platform for optimizing complex IT resources in a scalable manner, it makes cloud computing quite cost effective.

Virtualization is simply running multiple operating system and applications all together on the same server at the same time [10].

There are many virtualization tools that exist in the market. VMware is the biggest tool used for virtualization. Virtualization can also be done through Xen and Qemu in the Linux based OS. Other tools and techniques also exist to do virtualization. Virtualization for Cloud OS is done in the server part where multiple servers are needed to serve the request of the client. As multiple servers are needed virtualization is done on all the servers to run different OS on them. Now these OS's are responsible to respond to the client's request. The virtual machines which are made on the server can be configured of different physical memory. So now each Virtual machine is independently able to run the applications on the server side which in turn would be serving the client's request if it is called upon and set in action [9]. Other aspect of virtualization can be used in the IaaS module. The hardware can also be virtualized for the storage part. This storage of data is synced with the virtualized server which will be later discussed in the synchronization module.

\section{PROPOSED ALGORITHM}

Cloud computing resources are used efficiently to gain the maximum profits with the help of efficient load balancing algorithm which has been one of the Cloud computing service providers ultimate goals. Throttled load balancer maintains a record of the state of each VM (idle/busy). If a request arrives concerning the allocation of virtual machine or to use an application running on a virtual machine Load balancer sends the Id of VM to data center controller. Active monitoring Load balancer identifies the least loaded VM. In the proposed algorithm, the four major factors i.e., CPU Utilization, RAM Utilization, Memory Utilization and Network Utilization play a very important role in calculation of load for a specific VM.

The proposed algorithm is named as "Application Prioritized Load Balancer". In this algorithm, load is calculated based on the different types of applications provided. Different applications may have different priorities for CPU, RAM, Memory and Network Utilization. Let us define these priorities as $\operatorname{Pr}_{\mathrm{CPU}}, \operatorname{Pr}_{\mathrm{RAM}}$, $\operatorname{Pr}_{\mathrm{MEM}}$ and $\operatorname{Pr}_{\mathrm{NET}}$. From these priorities total of 32 combinations of priorities is possible depending upon the type of application used.

So the algorithm can be defined as:

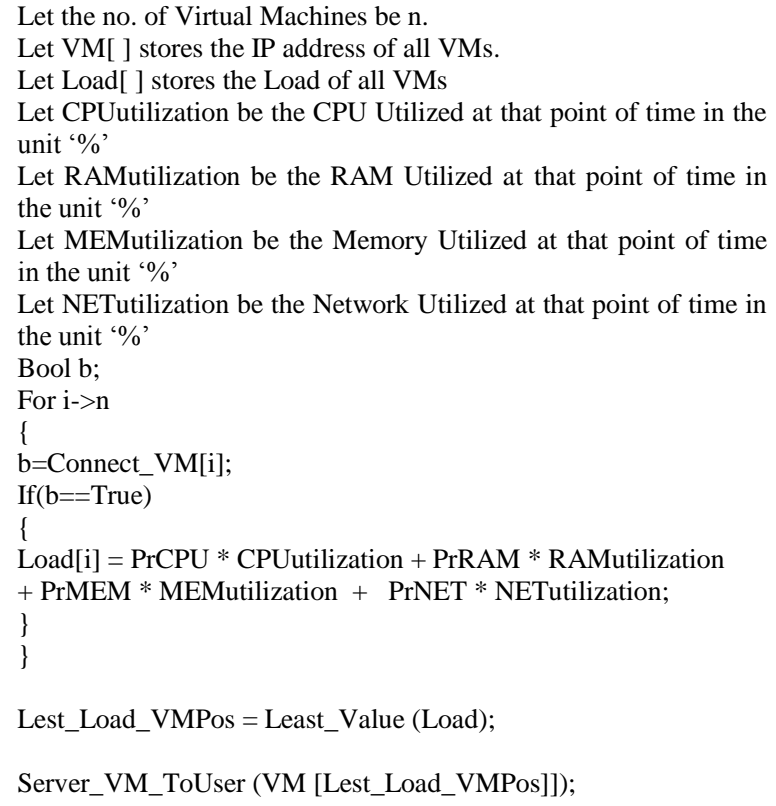

Let MEMutilization be the Memory Utilized at that point of time in the unit ' $\%$ '

Let NETutilization be the Network Utilized at that point of time in the unit ' $\%$ '

Bool b;

For i->n

\{

$\mathrm{b}=$ Connect_VM[i];

If $(b==$ True $)$

\{

Load $[\mathrm{i}]=\operatorname{PrCPU} *$ CPUutilization $+\operatorname{PrRAM} *$ RAMutilization

$+\operatorname{PrMEM} *$ MEMutilization $+\operatorname{PrNET} *$ NETutilization;

Lest_Load_VMPos $=$ Least_Value $($ Load $)$;

Server_VM_ToUser (VM [Lest_Load_VMPos]]);

For example: If we have a gaming cricket application that uses high graphics that user wants to use so we can say that $\operatorname{Pr}_{\mathrm{CPU}}>\operatorname{Pr}_{\mathrm{RAM}}>\operatorname{Pr}_{\mathrm{NET}}>\operatorname{Pr}_{\mathrm{MEM}}$

So, let us assume that

$$
\operatorname{Pr}_{\mathrm{CPU}}=4, \operatorname{Pr}_{\mathrm{RAM}}=3, \operatorname{Pr}_{\mathrm{NET}}=2, \operatorname{Pr}_{\mathrm{MEM}}=1
$$

So,

$$
\begin{gathered}
\text { Load }=4 * \mathrm{CPU}_{\text {utilization }}+3 * \mathrm{RAM}_{\mathrm{utilization}}+1 * \\
\mathrm{MEM}_{\text {utilization }}+2 * \mathrm{NET}_{\text {utilization }} ;
\end{gathered}
$$

Hence the Load for different types of application will vary, providing better accuracy and results. Therefore User will be able to use the application with good quality experience resulting in efficient resource allocation

\section{A. Geo-Replication Data Storage}

With the increase in the use of Cloud users the data is also increasing in a tremendous rate which makes it difficult to store and handle it safely. To provide the user a good experience of using the applications and scalability, use of data replication is very necessary. Data replication means, placing multiple copies of data on different servers so that it is safe and survive to any kind of failures. Secondly, the data copy is placed closer to the user so that data can be accessed with smaller latency. But now the point is, why replicating the data to all the servers? It causes a lot of redundancy and wastage of space on the server by storing the same data. Applications are being more focused on performance factor because an increase in latency results in loss of percentage of the user. It is becoming a key factor to find a data storage protocol that offers both consistency and efficiency. Thus, a better idea is being proposed to store the data on the server according to the user's geo-graphic location. 


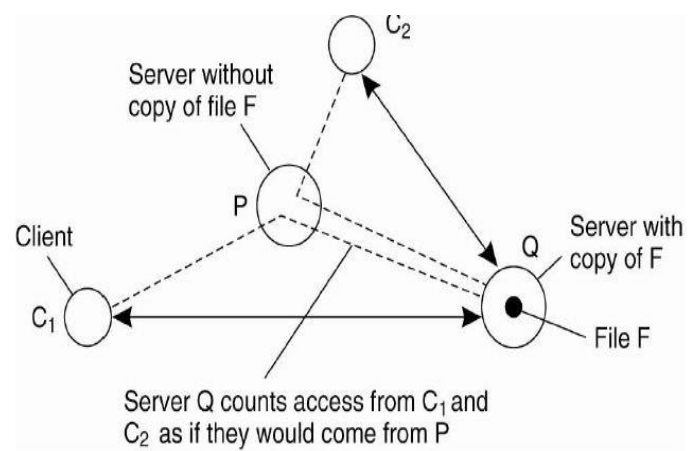

Fig.1. Server Initiated Replicas

Algorithm/Approach Proposed:

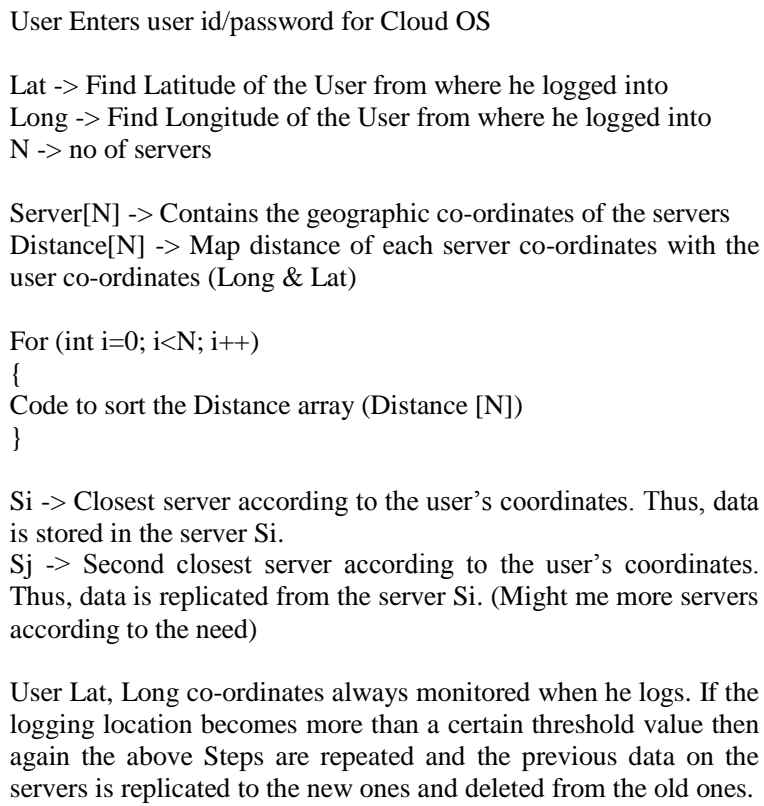

User Lat, Long co-ordinates always monitored when he logs. If the logging location becomes more than a certain threshold value then again the above Steps are repeated and the previous data on the servers is replicated to the new ones and deleted from the old ones.

Note: Everything will be based on the email_id of the user which is to be mapped with the servers and then change gradually.

Now, when the user requests for the data then the data will be retrieved using the same load balancing approach as discussed before between the servers where the data is stored and thus retrieved. The priority set for it will be Network then CPU then RAM and then Memory This in total reduces the redundancy and latency of the data storage system module.

\section{PRoposed ARCHITECTURE FOR ClOUd OS}

The architecture proposed for Cloud OS is made taking into consideration the virtualization, load balancing, Cloud storage, WebTop, and synchronization models in it. The whole architecture [15] is set up so that there is a smooth functioning of the client's request from his WebTop to the load balancer and then there is a proper sync of his data on the cloud with all the servers and also the requests are served by the VM's of each server in order to maintain the load of the server side VM's. The load balancer is shown in order to see that the resources such as CPU and memory are being efficiently shared among the VM's.

Now let's discuss the flow of the architecture shown:

(The basic requirement of using the Cloud OS is having an internet connection and a web browser.)

1. A user visits the cloud OS webpage through the browser and logs into the cloud OS.

2. The Webtop of the user is logged in where all the application icons, his dashboard and cloud drive will be shown.

3. Now when these applications are double clicked then the application event is triggered and the application request is forwarded to the load balancer and the load balancer decides which VM will serve the request based on the algorithm chosen for load balancing for efficient resource sharing.

4. When the Geo-Replicated cloud drive is opened then the server which is closest to the user's location comes in play and the Server with the least load holding the data of the user serves the request and opens the cloud drive running on the VM.

5. If the nearest Cloud storage server is fully loaded as discussed in point $\mathrm{E}$ above then the next server nearest to user holding the data serves the request.

6. All the Geo-Replicated data storage servers are in sync with each other.

7. Hence user can use all the applications smoothly and can access his personal cloud drive in a very short time.

8. After finishing his work on Cloud OS, the user can log out from the Cloud Webtop and next he logs in he gets his OS as left last time

\section{ANALYSIS AND RESULT}

Load balancing over application servers and Geo Replication over storage servers plays a very important role in making the system more efficient, scalable, reliable and robust. The following analysis shows that the proposed schemes for the two are better and more profitable

\section{Application Prioritized Load Balancing over} Application Servers

Results were recorded for comparing the proposed scheme with basic load calculation algorithm when creating 8 identical VMs with following specifications:

- $\quad$ RAM : 2 GB

- HARD DISK STORAGE SPACE: $50 \mathrm{~GB}$

- HOST OS: Windows 7

On using Load Runner as a tool for generating Load on the system for testing 100 users and getting Response time of applications for proposed as well as basic algorithm as following results were recorded 


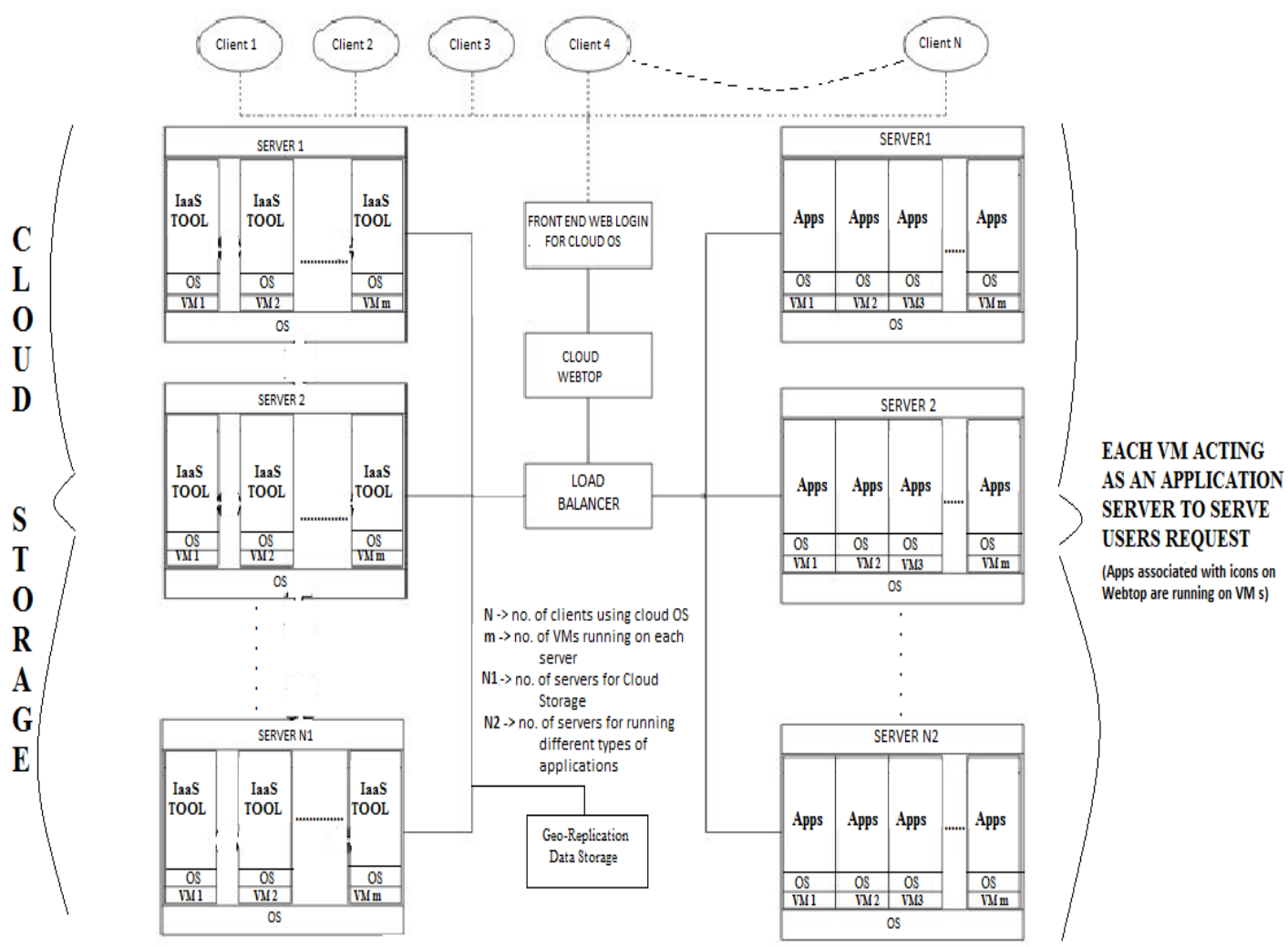

Fig.2. Architecture Proposed for Cloud OS

Table 1. Analysis for Basic Load balancing (CPU Utilization only) with Proposed Load Balancing Algorithm: Application Prioritization.(C-CPU, R-RAM, H-HARD DISK/MEMORY, N-NETWORK

\begin{tabular}{|c|c|c|c|}
\hline Application & $\begin{array}{c}\text { Priority of } \\
\text { Application } \\
\text { for Proposed } \\
\text { Algorithm }\end{array}$ & $\begin{array}{c}\text { Response } \\
\text { Time for } \\
\text { Old Basic } \\
\text { Algorithms }\end{array}$ & $\begin{array}{c}\text { Response } \\
\text { Time for } \\
\text { Proposed } \\
\text { Algorithms }\end{array}$ \\
\hline Cricket & CNRH & $0.9459 \mathrm{sec}$ & $0.7153 \mathrm{sec}$ \\
\hline Sudoku & RNCH & $0.2538 \mathrm{sec}$ & $0.1498 \mathrm{sec}$ \\
\hline Web Editor & NRCH & $0.2118 \mathrm{sec}$ & $0.1006 \mathrm{sec}$ \\
\hline Image Viewer & RCNH & $0.3162 \mathrm{sec}$ & $0.1979 \mathrm{sec}$ \\
\hline Music Player & CRNH & $0.3947 \mathrm{sec}$ & $0.2499 \mathrm{sec}$ \\
\hline $\begin{array}{c}\text { Snakes and } \\
\text { Ladders }\end{array}$ & RNCH & $0.2641 \mathrm{sec}$ & $0.1746 \mathrm{sec}$ \\
\hline Chess & CRNH & $0.4843 \mathrm{sec}$ & $0.3152 \mathrm{sec}$ \\
\hline Video Player & CRHN & $0.6179 \mathrm{sec}$ & $0.4872 \mathrm{sec}$ \\
\hline
\end{tabular}

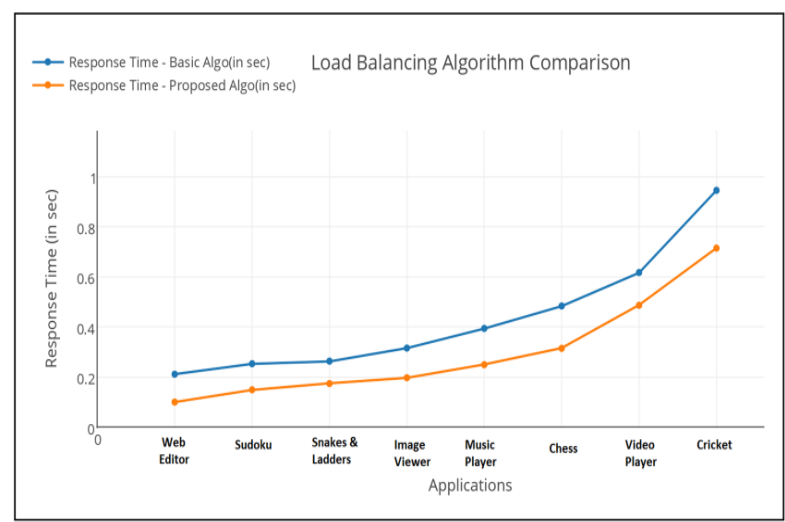

Fig.3. Comparison of Old Basic Algorithm based on CPU Utilization with proposed load balancing algorithm i.e. Application Prioritization
Hence the results clearly depict that Application prioritized load balancing algorithm have a lesser response time for same number users which compared for an application resulting in better efficiency and quality.

On doing analysis of the above stated applications for different number of users from 0 to 200, it was being discovered that applications having CPU as their first priority were having similar type of pattern on generating graph for response time vs number of users and applications having RAM as their first or second priority were having similar type of pattern. The patterns for both are:

\section{$\rightarrow$ Resporse Time - Basic Aligo(n sec) $\quad$ Pattern for CPU intensive application}

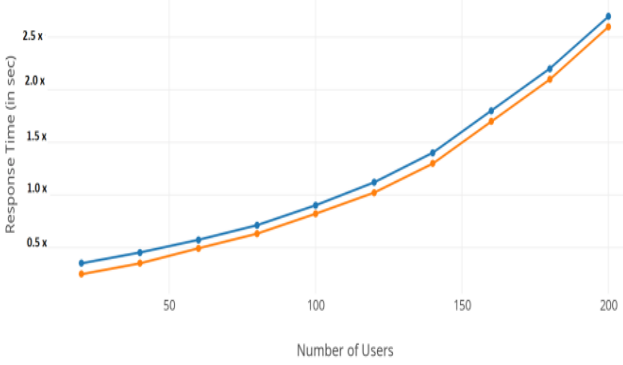

Fig.4. Pattern for CPU Intensive applications 
$\underset{\rightarrow-\text { Responsse Time - Proposesed Algog(in sec) }}{\rightarrow}$ Pattern for RAM intensive Applications

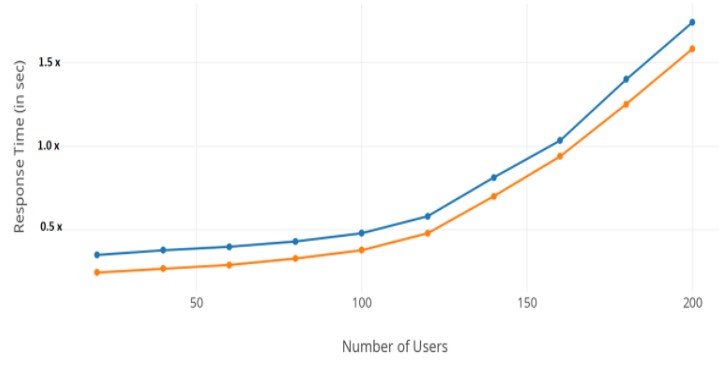

Fig.5. Pattern for RAM Intensive applications

\section{Geo Replication of Data over Storage Servers}

The Result of Geo-Replication on Data Storage Cloud Servers is very much evident in itself. Where the same data was being stored in $\mathrm{N}$ servers now because of georeplicating the data according to user's location, the data is stored in two or three servers. This reduces the redundancy of data storage space on servers.

The other advantage is that, as the data stored is always closer to the user's location thus the latency will be reduced as the data will be fetched among the least loaded server among the geo-replicated cloud servers.

\section{FUTURE DIRECTION}

- Though the architecture of the cloud OS is efficient but there are many issues which can be further improvised.

- The WebTop can be made more realistic to an actual OS if some drivers can be installed. Like a USB drive can be detected.

- Users can deploy their own applications on the OS.

- Providing more applications to the users on the WebTop.

- Load balancing techniques can be improvised further by keeping in consideration environment i.e. Centralized, Distributed or Semi- Distributed.

- Synchronization can be done.

- Efficient memory management techniques can be applied for efficient resource management.

- Process Scheduling can be done in a more efficient manner.

\section{CONCLUSION}

The Cloud OS aims to provide an array of applications which resides on the WebTop itself. The whole environment is that of the desktop which is simply running on a web browser. The best part is that one does not need his personal laptop every time with them as his personal pc is on cloud with the help of Cloud OS. The WebTop is ubiquitous. Along with this features of load balancing and geo-replication of data has been implemented.

The results of the proposed approach which is application prioritized load balancing clearly shows that the algorithm has lesser response time for the web applications than the normal approach. Secondly, when the data is geo-graphically replicated over the storage servers then the space is saved as redundancy is reduced over the storage servers and even the latency for the user to gets his data is reduced. Altogether this makes the Cloud OS system more robust, powerful and more efficient. Even the user is pleased to use the Cloud OS which is the new era of Cloud Computing

\section{REFERENCES}

[1] C.Eunjeong, B.Chang Seok and L. Jeunwoo "Data synchronization between adjacent user devices for personal cloud computing" Consumer Electronics (ICCE), 2012 IEEE International Conference, pp. 49-50, Jan 2012

[2] Chandra D.G and Malaya D.B, "A Study on Cloud OS " Communication Systems and Network Technologies (CSNT), 2012 International Conference, pp. 692-697, May 2012

[3] C.Jun, W.Xing, Z.Shilin, W.Zhang and N.Yanping, "A Decentralized Approach for Implementing Identity Management in Cloud Computing" Cloud and Green Computing (CGC), 2012 Second International Conference, pp.770-776, 1-3 Nov. 2012

[4] Y.Hyun-Suk, Yvette E. Gelogo and K.J.Kyung, "Securing Data Storage in Cloud Computing", Journal of security engineering, pp. 251-260, 2012

[5] Mladen A. Vouk, "Cloud Computing - Issues, Research and Implementations" Journal of Computing and Information Technology - CIT, pp. 235-246, 2008

[6] G.Jun-wei, Dying-long and F.Yi-qiu , "Research on Storage Virtualization Structure in Cloud Storage Environment,"Multimedia Technology (ICMT), 2010 International Conference, Osaka, pp. 1-4, 19-23 April 2010.

[7] P.Fabio, B.Peter, D. Alessandro, J. Nico, S. Thanos and S.Moritz, "Towards a Cloud Operating System", Network Operations and Management Symposium Workshops (NOMS Wksps), 2010 IEEE/IFIP, pp.335 - 342, 19-23 April

[8] S.Prof.Meenakshi and S.Pankaj,"Performance Evaluation of Adaptive Virtual Machine Load Balancing Algorithm", International Journal of Advanced Computer Science and Applications, Vol. 3, No.2, 2012, pp. 86-88, 2012.

[9] R.Haozheng, L.Yihua and Y.Chao, "The load balancing algorithm in cloud computing environment", Computer Science and Network Technology (ICCSNT), 2012 2nd International Conference, pp.925 - 928, 29-31 Dec. 2012.

[10] X.Yuping, Z.Yongzhao, "Virtualization and Cloud Computing" , Future Wireless Networks and Information Systems, Lecture Notes in Electrical Engineering Volume 143, pp 305-312, 2012.0

[11] P.Stuedi, I.Mohomed and D.Terry, "WhereStore: Location-based Data Storage for Mobile Devices Interacting with the Cloud" Proceedings of the 1st ACM Workshop on Mobile Cloud Computing \& Services: Social Networks and Beyond in New York, USA, 2010.

[12] Dahnert, A. "HawkEyes: An advanced IP Geolocation approach: IP Geolocation using semantic and measurement based techniques" Cybersecurity Summit (WCS), 2011 Second Worldwide in London, pp. 1-3, 1-2 June 2011.

[13] Lin, G.; Deze, Z.; Peng, L.; \& Song, G., "Cost Minimization for Big Data Processing in Geo-Distributed Data Centers" Emerging Topics in Computing, IEEE 
Transactions, Volume PP, Issue 99, 11 March 2014

[14] Dinh, Hoang T., et al. "A survey of mobile cloud computing: architecture, applications, and approaches." Wireless communications and mobile computing. Vol. 13.18, PP. 1587-1611, 2013

[15] Kim, Won. "Cloud computing architecture." International Journal of Web and Grid Services Vol. 9.3, PP. 287-303, 2013

\section{Authors' Profiles}

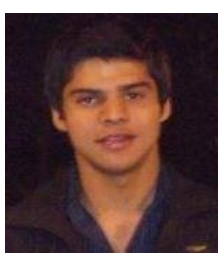

Gaurav Chhatwal obtained his Bachelor's Degree in Computer Science and Engineering and Master's Degree in Computer Science and Engineering with Major in Cloud Computing from Jaypee Institute of Information Technology, Noida, India. He is a Oracle Certified Java SE 6 Professional Programmer. He has worked on various projects like Cloud WebTop with PC sync Client Application using Java and PHP; Autonomous Semi-Distributed Load Balancing in Cloud Computing using Java and PHP; Controlling PC via speech jn Java; RSA based cryptography for distributing files over a network and Remote Desktop Application- Controls PC through Mobile via Bluetooth using Java. He has won many programming competitions at college level. $\mathrm{He}$ is currently working at Othain Group as a Software Engineer for past 2.5 years.

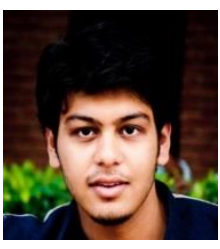

Rishabh B. Shah, obtained his Bachelor's Degree in Computer Science and Engineering and Master's Degree in Computer Science and Engineering with Major in Cloud Computing from Jaypee Institute of Information Technology, Noida, India.He has worked on various projects like Cloud WebTop with PC sync Client Application using Java and PHP; Geo Replication of Data Over Cloud Storage service using Java and PHP; Proxy Server Implementation in Python; RSA based cryptography for distributing files over a network and WebTV using PHP, JavaScript. He has won many programming competitions at college level. He is currently working at Othain Group as a Software Engineer for past 2.5 years.

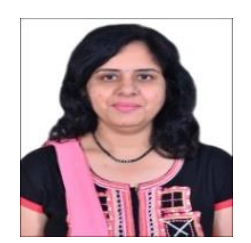

Kavita Pandey, received her B.Tech. in Computer Science and Engineering from M.D. University in 2002 and M.Tech. (CS) from Banasthali Vidyapeeth University in year 2003. She has obtained her Ph.D. (CS) from Jaypee Institute of Information Technology, Noida, India in January, 2017. She is currently working as an Assistant Professor in JIIT, Noida. Her research interests include Mobile Ad hoc Networks, Vehicular Ad hoc Networks, Optimization Techniques and Network Security. She has published various papers in International journals and conferences including Wiley, IEEE, Springer, Inderscience, etc.

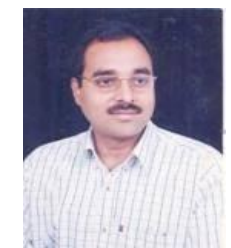

Prakash Kumar is currently an Associate Professor in Jaypee Institute of Information Technology,(Deemed to be University), Noida, India. His area of interest is Operating Systems, Distributed and Cloud Computing, Virtualization Technology and Internet of Things. He has been awarded $\mathrm{Ph}$ $\mathrm{D}$ in the field of Cloud Computing, viz. Resource allocations using virtualization in Cloud environment. He has received his M. Tech in Computer Science and Technology from University of Roorkee (Now Indian Institute of Technology, Roorkee), India. He has published many papers in International journals and conferences.

How to cite this paper: Gaurav Chhatwal, Rishabh Shah, Kavita Pandey, Prakash Kumar, "Developing Cloud OS", International Journal of Information Technology and Computer Science(IJITCS), Vol.10, No.4, pp.26-32, 2018. DOI: 10.5815/ijitcs.2018.04.03 